\title{
Sleep Hygiene and its Related Factors Among the Elderly in Tabriz, Iran
}

\author{
Soheila Bani ${ }^{1}$, Shirin Hasanpour ${ }^{1 *}$, Jamileh Malakuti ${ }^{1}$, Parvin Abedi ${ }^{2}$, \\ Somayeh Ansari ${ }^{2}$
}

\section{Article History:}

Received 27 November 2013

Accepted 3 February 2014

Revised 15 January 2014

Available online 4 February 2014

\section{Keywords:}

Elderly

Environmental Factors

Nutritional and Pharmaceutical

Factors

Sleep Hygiene

\section{Corresponding Author:}

Shirin Hasanpour, Tabriz University of Medical Sciences,

Tabriz, Iran.

Tel: +984114796770

Email:

shirinhasanpoor@yahoo.com

\begin{abstract}
Objectives: The aim of this study was exploring sleep hygiene and its related factors among the elderly in Tabriz, Iran. This study was conducted on 100 elderly men and women over the age of 60 in Tabriz, Iran.

Materials and Methods: This was a cross-sectional study which conducted on 100 men and women over 60 years old who were selected among four active retirement centers in Tabriz, Iran in 2010. A questionnaire consisting of four sections was used for data collection. Data was gathered on socio-demographic, personal, environmental and nutritional- pharmaceutical factors of sleep hygiene. Overall sleep hygiene was considered to be as optimal if the score of three domains was between 119 and 177, moderate with score 60-118 and unfavorable with score 0-59. Descriptive statistics and chi-square tests were used to analyze the data.

Results: Most participants (74\%) claimed that they wake up frequently during the night. Sleep hygiene in $16 \%$ of the participants was optimal and in $84 \%$ was moderate. Regarding personal, environmental and nutritional- pharmaceutical factors, sleep hygiene in $18 \%, 16 \%$ and $54 \%$ of the samples was optimal respectively. There was a significant relationship between gender, marital status, number of household, the amount of revenue, marital life satisfaction with sleep hygiene in the elderly $(\mathrm{p}<0.05)$.

Conclusion: The findings revealed that only small number of participants enjoyed sleep hygiene. Considering the optimal impact of sleep hygiene and its importance in fixing sleep disorders, improving sleep hygiene through educational programs can decrease the sleep disorders and improve the quality of life.
\end{abstract}

1 - Tabriz University of Medical Sciences, Tabriz , Iran.

2- Jundishapur University of Medical Sciences, Ahvaz, Iran. 


\section{Introduction:}

Human need sleep just like food and water. Adults need 7-8 hours sleep at night to have a normal physiologic function during the day (1). When people get older, they need less sleep than younger adults, as the elderly, in their 70s, need 30-60 minutes less than adults in their 20s (2). The quality of night sleep decreases with age and 30\% of the elderly may experience chronic insomnia (3). A study in Sweden showed that; $70 \%$ of the elderly had sleep problems and 62 had some experience of pain during previous 4 weeks. Sleep problems and pain were more frequent among women than men (4). According to a study conducted in the Kahrizak Nursing Home in Tehran, Iran, 303 (39.2\%) of the elderly, including 86 (34.7\%) men and 217 (41.1\%) women had insomnia syndrome (5).

Sleep hygiene means improving behavioral and environmental factors that ameliorate sleep. A good sleep at night can promote daytime alertness (6).

Educating the elderly about age-related changes in sleep pattern and good sleep hygiene may be useful treatment for many elderly to overcome their insomnia. Maintaining a regular sleep and wake-up time, decreasing daytime naps, increasing physical activity and avoiding heavy meals, alcohol and coffee consumption are examples of sleep hygiene (7).

Having a restful sleep can increase the life span, and one of the best ways to achieve a restful sleep in the elderly is sleep hygiene. In fact, sleep hygiene is a set of personal habits that determines the sleep quality. The amount of sleep also has a great impact on health and wellbeing (8). A review by Dollander showed that; environmental factors, such as working constraints and behavioral factors, e.g. going to bed too early, to read or to look at TV when going to bed, could decrease sleep quality. Furthermore, regular intellectual activities close to bedtime, having a late meal in the evening, noisy or unhealthy environment, physical hyperactivity or sleeping after lunch; and finally psychiatric factors, such as a major depressive episode could increase sleep disorders (9). Sleep disorder is not a disease but maybe a symptom of physical and mental damage. In some people, sleep disorders tend to increase according to their behavior, life style and other factors (10). According to medical results, sleep disorders in the elderly can cause anxiety, sleepiness, unconsciousness, intellectual damage, delirium, slow psychomotor and increased accident risk and injury. These symptoms may even jeopardize the quality of life and cause an enormous social and economic burden on the health system (11). The quality of life is affected by the loss of consolidation and depth of sleep, especially in older people (12). A study by Reid et al showed that aerobic physical activity with sleep hygiene education could improve the sleep quality, mood and quality of life in older adults with chronic insomnia (13).Considering that more than $50 \%$ of the elderly suffer from sleep disorders; health care providers need to endeavor to treat these disorders (14). Using behavioral or psychotherapeutic approaches is preferable to medication for treating sleep disorders (15). There is a lack of sufficient information about sleep hygiene and related factors in the Iranian elderly. The primary aim of this study was to determine sleep hygiene and its related factors among the elderly in Tabriz, Iran.

\section{Material \& Methods:}

This was a cross-sectional study, which investigated the sleep hygiene and related factors among the elderly in Tabriz, Iran, 2010. The study design was approved by the Ethics Committee of the Tabriz University of Medical Sciences (code: 91152).

The study sample consisted of 100 men and women over 60 years of age who were selected from four active retirement centers in Tabriz (Education department, Power department, Armed forces and Health and Treatment office) using the census sampling method based on eligibility criteria of study. The total numbers of retired elderly who were the member of retirement centers was 476.The inclusion criteria were; more than 60 years of age and membership in one of the specified retirement centers. The exclusion criterion was using sleeping pills. We phoned150 elderly who had inclusion 
criteria for the study (75 women and 75 men).Fifty elderly could not complete the study because of; 15 were sick, 12 had plan to take a trip and 23 started to use sleeping pills, finally, 100 elderly (50women and 50 men) completed the study. All participants signed an informed written consent prior to the study. Almost all of the participants lived with their families.

\section{Measures :}

The research instrument in this study was a self regulated questionnaire that was completed by every participant in the study. The questionnaire consisted of four parts. The first part included 11 questions about the socio-demographic characteristics of the subjects. The second part consisted of 19 questions about personal factors relating to sleep hygiene in which the results were classified as optimal (39-57), moderate (2038) and unfavorable (0-19). Statements such as "I have a regular sleeping time", I wake up every day in a certain time" and "I wear comfortable cloths when sleeping" were included in this section. The third part included 23 questions about environmental factors relating to sleeping hygiene and the results were classified as optimal (47-69), moderate (24-46) and unfavorable (023).Statements like; "my bedroom has a window, and sometimes I open the window to change the weather", " my mattress is not too hard not too soft" were included in this part of questionnaire. The final part included 17 questions about nutritionalpharmaceutical factors pertaining to sleep hygiene, which were conventionally determined as optimal (35-51), moderate (18-34) and unfavorable (0- 17). The following are examples of statements in this area: "I have a regular diet", "I avoid drinking too much fluids during night". Overall the sleep hygiene in three domains was considered to be as optimal (119-177), moderate (60-118) and unfavorable (059).The validity and reliability of the questionnaire were assessed using the content validity and test-retest. Ten elderly completed the questionnaire, and after 10 days they requested to answer the same questions, that they answered 10 days ago.
The correlation coefficient between the two completed questionnaires was $85 \%$.

The findings were analyzed using SPSS softwarever13. The descriptive statistics and Chi-square test were used for analyzing data.

\section{Results:}

According to the results of this study, the mean age of the subjects was 69.5. The majorities of the subjects were married and had primary school literacy. Most of them received 200-400 Tuman (Iranian currency) pension. The majority of the subjects (92\%) were living with their families with an average number of 2.56 persons. Most participants (74\%) claimed that they wake up frequently during the night, and 36\% were satisfied with their marital life.

Overall, in $16 \%$ of the subjects, sleep hygiene was optimal, and, in $84 \%$, it was at the moderate level. In relation to personal factors, the sleep hygiene in $18 \%$ of the subjects was optimal and $82 \%$ of them were moderate. Regarding the environmental factors, the sleep hygiene in $16 \%$ and $84 \%$ of participants was at the optimal and moderate level, respectively. Regarding the nutritional-pharmaceutical factors, the sleep hygiene in $54 \%$ of the subjects was optimal, and in $46 \%$, it was moderate (Fig. 1).

Considering the personal factors of sleep hygiene, $32 \%$ of the subjects claimed that they did not smoke before bedtime, they certainly brush their teeth and read something (28\%) and wake up at a certain time every morning (40\%). However a small percentage of them left their room if they could not fall asleep for more than 30 minutes (9\%) exposed to the sunlight during the daytime (4\%) and had a normal weight (8\%).

In respect of the environmental factors of sleep hygiene, the majority of the subjects used their beds just for sleeping (41\%), kept their room dark or used eye patches (37\%). However, a small percentage of them kept their room temperature between 1718degrees Celsius (9\%), changed their room occasionally (4\%) and turned their mattress once a week (5\%).

Regarding the nutritional-pharmaceutical factors of sleep hygiene, the majority of the 
subjects avoided the arbitrary use of sleeping pills (41\%), had a light dinner (41\%) and did not use soft drinks before bedtime (37\%). A small percentage of them ate an apple before bedtime (5\%), drank milk and honey (8\%) and avoided tea or coffee before bedtime ( $9 \%)$.

Female were reported significantly better sleep hygiene than that in men $(\chi 2=7.40$, $\mathrm{p}=0.005)$. Elderly with moderate revenue had better sleep hygiene $(\chi 2=24.2$, $\mathrm{p}<0.001)$. The number of household was significantly less in the people with optimal sleep hygiene than that in the moderate sleep hygiene $(75 \%$ vs. 33\%, $\chi 2=9.93$, $\mathrm{p}=0.02$ ) (Table 1).

\section{Discussion:}

The findings of the study suggested that a small number of old people admitted the optimal level of sleep hygiene. Nobahar's study in Iran confirms these findings (16). The present study indicated that the elderly tended to follow nutritional and pharmaceutical factors more than environmental factors. These results are not in line with other researchers, as Brumen in 1996 found that $20.1 \%$ of French people use sleeping pills or tranquilizers (17).

Regarding personal factors, the majority of participants were waking up at a certain time. A human's body has a circadian rhythm that is generated and maintained endogenously by the suprachiasmatic nucleus (SCN) in the hypothalamus which is also called the timekeeper in the body. This endogenous body clock is affected by a number of exogenous stimuli, e.g. light. Many physiological processes, such as body temperature, sleep and alertness are governed by the 24 hour clock (18).

According to the results of this study, only a small percentage of the elderly avoided tea or coffee before bedtime. Considering the Iranian's eating habits, generally and the people of Azerbaijani (the province including the location of the study, Tabriz) in particular, who are interested in drinking tea after their meals, it seems that educating old people, particularly regarding changing their eating habits, can be beneficial for sleep hygiene. A study with the aim of contributing to sleep quality in the elderly who lived in a retirement community in Israel showed that when the stability of daily routine increased (measured by Scale of Older Adults Routine) it could predict shorter sleep latency; higher sleep efficiency and improvement of sleep quality (19).For the elderly who live in the retirement community, it will be easier to change their lifestyle; However, almost all participants in our study lived with their families and may need more time to change their behavior.

In a study Wolkove and his colleagues(2007), concluded that education for the elderly and focusing on this issue have played a basic role in the reduction of the complaints over sleep disorders (20). Poor sleep hygiene, such as daytime napping, bedroom environment, bed partner, dietary habits or lack of physical activity can be an initiator for sleep disorders (21).

The results about the environmental factors showed that only a small percentage of the elderly kept their room temperature between 17-18 degrees Celsius and most of them tended to keep their houses warm, especially during winter. Only a small percentage of them changed their room occasionally or turned their mattresses once a week. Gooneratne et al (2011) was conducted a cross-sectional study on 242 older adults to investigate the sleep parameters using the Pittsburgh Sleep Quality Index and sleep treatment option. The results showed that participants used different regimens to improve their sleep. The most common interventions were watching $\mathrm{TV}$ or listening to the radio $(66.4 \%)$ and reading (56.2\%). Using sleeping pills had the greatest self-reported effectiveness (22). In our study, most of participants (74\%) claimed that they wake up frequently during the night, but they did not seek any logical solutions for their problem.

Among the personal factors, a small percentage of the elderly left their room, if they could not fall asleep for more than 30 minutes, had exposure to sunlight during the day and had a normal weight. Changing lifestyle, such as doing suitable exercises on sunny days, sitting or walking in a nearby 
park and also having a good diet plan that will prevent the elderly from gaining weight, can improve sleep hygiene. Results of a study conducted by Hock et al (2001), revealed that the elderly who received sleep hygiene instructions for eight weeks showed considerable improvement in their sleep hygiene condition(23).

A qualitative study conducted by Roland (2011) to assess the sleep disturbance among the elderly in a nursing home showed that non-pharmacological intervention, such as sleep restriction, scheduled bright light, bed massage and physical activity led more sleep improvement among the elderly(24). The studies of Bloom and colleagues in 2009 indicate that behavioral changes are very useful in treatment of sleep disorders and the improvement of sleep hygiene. Some wrong behaviors that may cause sleep disorders include late time exercise, drinking tea and coffee or alcoholic beverages before bedtime, frequent naps during the day, anxiety resulting from insomnia, watching exciting movies before bedtime, heat, extreme light and noise and staying in bed for a long time (25). The present study showed that there were only 16 individuals whose sleep hygiene was optimal. The emphasis on teaching changes of lifestyle is easily affordable from different aspects like cost and time rather than the treatment of sleep disorders, and it also helps to improve the quality of life.

The results of our study revealed that female had significantly better sleep hygiene than men. In a study by Habte-Gabr et al (1991) on 3097 persons aged 65 years and older, the results showed that sleep patterns were significantly related to educational attainment, life satisfaction, self- perceived health status and physical function status. They also found that women had longer sleep latency and fewer hours of sleep than men (26). Our results are in line with HabteGaber et all's study in addition, in our study, sleep hygiene in women was significantly better than in men.
Our results showed that the elderly with marital life satisfaction had more optimal sleep hygiene. Healthy relationship may improve sleep pattern, by inducing feeling of safety and security (27).

A study conducted by Tel(2013) with aim of evaluating sleep quality and quality of life among elderly showed the significant relationship between gender, marital status, educational status, the person with whom the elderly lived, physical disorders with sleep quality and quality of life (28).

Strengths and limitations :

There is no exclusive information about sleep hygiene among the elderly in Iran. The results of this study can provide policy makers some basic information about sleep patterns for the elderly.

We could not recruit the elderly out of retirement centers because of the difficult access to other seniors in society; therefore, the results of this study cannot be generalized to the entire population.

Furthermore, we did not assess the chronic diseases associated with aging that may cause insomnia in the elderly. A study by Foley et al, (2004) showed that sleep disorders in older adults are often secondary to their chronic diseases, e.g. heart disease, depression and chronic body pain (29).

\section{Conclusion :}

The findings revealed that only small number of participants enjoyed sleep hygiene. Considering the optimal impact of sleep hygiene and its importance in fixing sleep disorders, improving sleep hygiene through educational programs can decrease the sleep disorders and improve the quality of life.

\section{Conflicts of interest:}

Authors declare that there is no any conflict of interest.

\section{Acknowledgments:}

We hereby, would like to thank all those who have helped us with this research, especially the honorable authorities of the retirement centers and the reverend seniors in Tabriz 
Figure 1. Distribution of participants according to personal, environmental and nutritionalpharmaceutical factors.

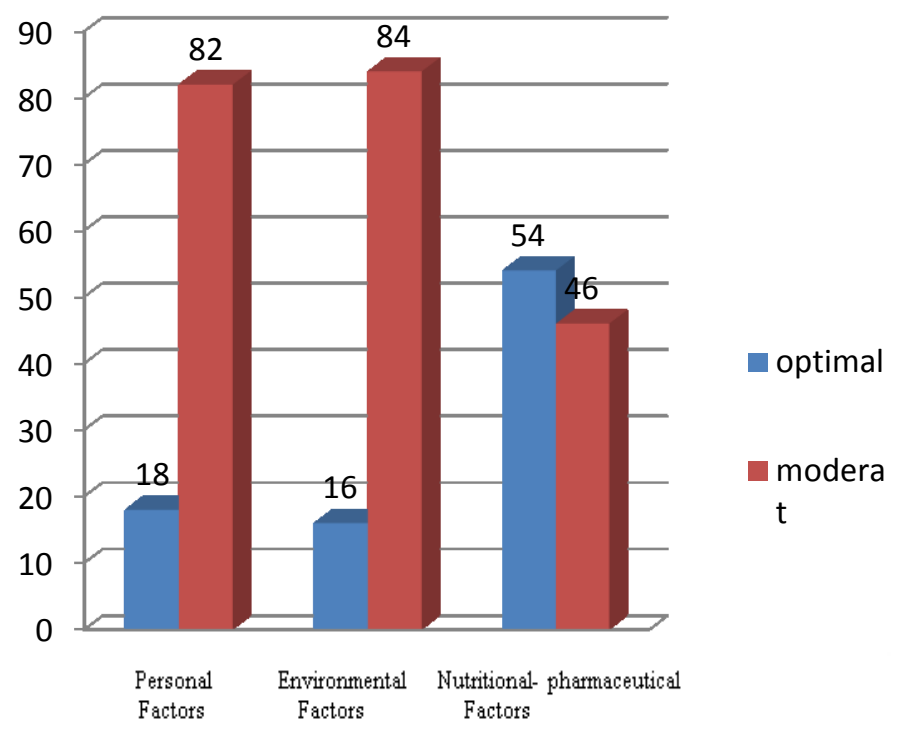

Table 2 The relationship between sleep hygiene and some socio-demographic characteristics.

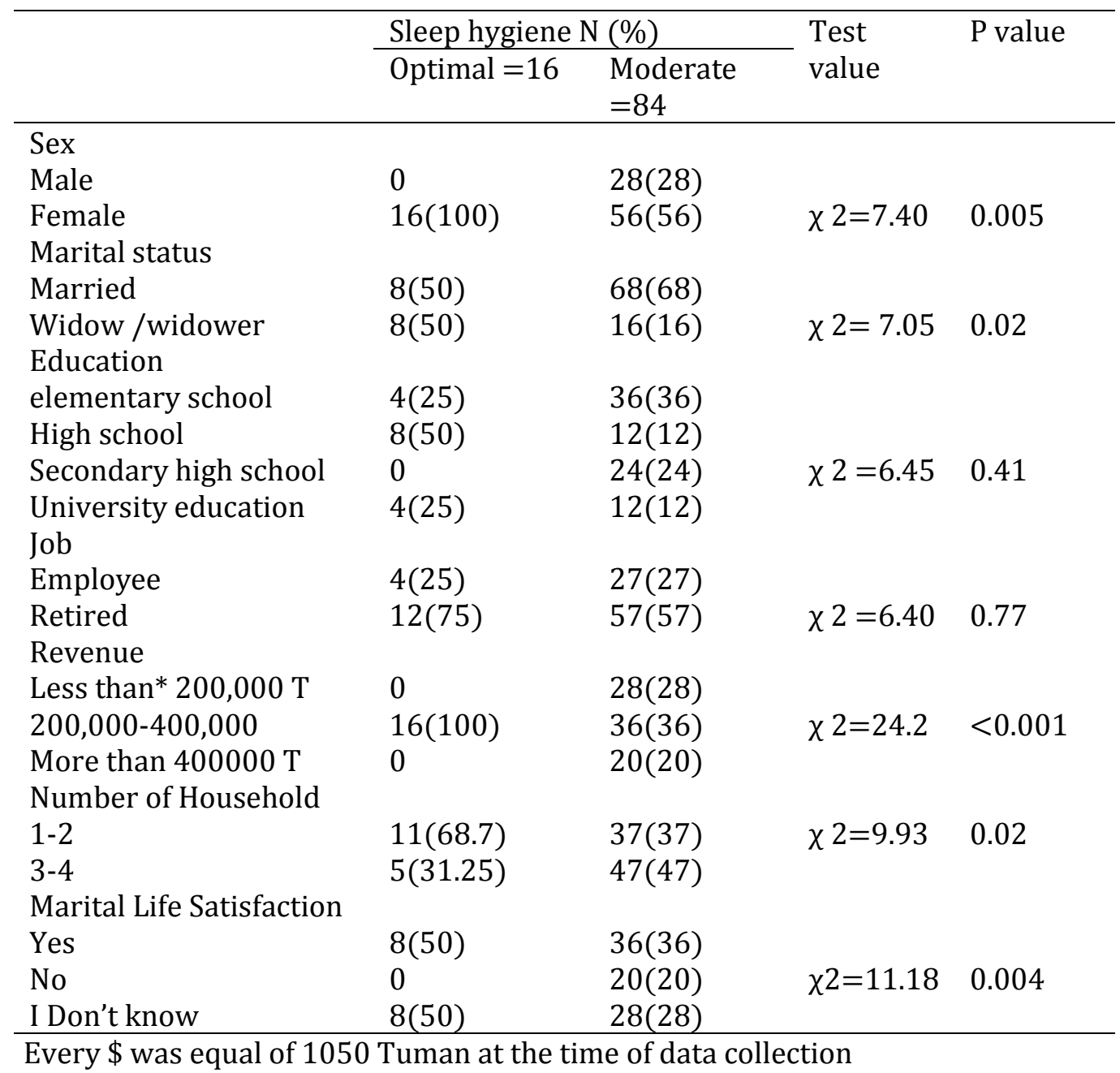




\section{References:}

1. Goolsby M. AANP insomnia surveys: sleep uncovered. J Am Acad Nurse Pract. 2006; 18 (12):557-558.

2. Harbison J. Sleep disorders in older people. Age Aging. 2002; 31 (suppl 2):6-9.

3. Hood B, Bruck D, Kennedy G. Determinants of sleep quality in the healthy aged: the role of physical, psychological, circadian and naturalistic light variables. Age Aging. 2004; 33(2):159165.

4. Lindstrom V, Andersson K, Lintrup M, Holst G, Berglund J. Prevalence of sleep problems and pain among the elderly in Sweden. J Nutr Health Aging. 2012; 16(2): 180-3.

5. Mousavi F, Tavabi A, Iran-pour E, Golestan B. Prevalence and associated factors of insomnia syndrome in the elderly residing in Kahrizak Nursing Home, Tehran, Iran. Iran J Public Health. 2012; 41(1):96-106

6. Van der Heijden K.B, Smits M.G, Gunning W.B. Sleep hygiene and actigraphically evaluated sleep characteristics in children with ADHD and chronic sleep onset insomnia. J Sleep Res.2006; 15(1): 55-62.

7. Cheng S.K, Dizon J. Computerised cognitive behavioural therapy for insomnia: a systematic review and meta-analysis.PsychotherPsychosom. 2012; 81(4):206-16.

8. Edward K, Wang Y. Adequate sleep among adolescents in positively associated with health status and health - related behaviors.BMC Public Health. 2006; 6:59.

9. Dollander M. Etiology of adult insomnia.Encephale. 2002; 28(6):493-502.

10. Christa J, Jahchens B. Sleep disturbances\& Healthy sleep .North America:Awsna publication. 2004; P:3-4.

11. Lavie P. Insomnia and sleep disordered breathing. Sleep Med. 2007; 8 Suppl 4:S21-5.

12. Wauquier A, Van Sweden B, Lagaay A.M, Kemp B, Kamphuisen H.A. Clinical investigation: ambulatory monitoring of sleep-wakefulness patterns in healthy elderly males and females (>88 years): the "senieur" protocol. J Am GeriatrSoc. 1992; 40: 109-114.

13- Reid K.J, Baron K.G, Lu B, Naylor E, Wolf L, Zee P.C. Aerobic exercise improves selfreported sleep and quality of life in older adults with insomnia. Sleep Med. 2010; 11(9): 934940.

14- Kamel N.S, Gammack J.K. Insomnia in the elderly cause approach, and treatment. Am J Med. 2006; 119(6):463-9

15- Lande R.G, Gragnani C. Nonpharmacologic approaches to the management of insomnia. The Journal of the American Osteopathic Association. 2010; 110 (12), 695-701. 
16- Nobahar M, Vafayi A. The survey of various sleep disorders and ways to elimination of them in elderly. The Iranian Journal of geriatric. 2007; 2(4):263-7. (Persian)

17- Bruman J.E, Lundn L.G, Hetta J. Insufficient sleep in the general population. NeurophysiolCli. 1996; 26(1):30-9

18- Boivin D.B, Czeisler C.A, Dijk D.J, Duffy J.F, Folkard S, Minors D.S, Waterhouse J.M. Complex interaction of the sleep wake cycle and circadian phase modulates mood in healthy subjects. Arch Gen Psychiatry. 1997; 54:145-52.

19- Zisberg A, Gur-Yaish N, Shochat T. Contribution of routine to sleep quality in community elderly. Sleep. 2010;33(4):509-14.

20- Wolkove N, Elkholy O, Baltzan M, Palayew M. Sleep and aging. CMAJ. 2007; 176(10):1449-54.3.

21- Zarcone V.P. Sleep hygiene. 2nd ed. Philadelphia: WB Saunders. 1994; 542-6.

22- Gooneratne N.S, Tavaria A, Patel N, Madhusudan L, Nadaraja D, Onen F, Richards K.C. Perceived effectiveness of diverse sleep treatments in older adults. J Am GeriarSoc. 2011; 59(2): 297-303.

23. Hoch C.C, Reynolds C.F, Buysse D.J, Monk T.H, Nowell P, Begley A.E, Dew M.A. Protecting sleep quality in later life: pilot study of bed restriction and sleep hygiene. Journal of Gerontology: Psychological Science. 2001; 56B (1): 52-59.

24. Roland N. Sleep disturbances among elderly people in Nursing home: A nonpharmacological approach. Dissertation on Human ageing and elderly services. Sleep.2011; 43 (5):61-8

25- Bloom H.G, Ahmed I, Alessi C.A, Ancoli-Israel S, Buysse D.J, Kryger M.H, Zee P.C. Evidence-Based Recommendations for theassessment and management of sleep disorders in older persons. J Am GeriatrSoc. 2009; 57(5):761-789.

26- Habte-Gabr E, Wallace R.B, Colsher P.L, Hulbert J.R., White L.R,Smith I.M. Sleep patterns in rural elders: demographic, health, and psychobehavioral correlates. J ClinEpidemiol. 1991; 44(1):5-13.

27- Troxel W.M, Robles T.F, Hall M, Buysse D.J. Marital quality and the marital be: examining the covariation between relationship quality and sleep. Sleep Med Rev. 2007; 11(5): 389-404.

28- Tel, H. Sleep quality and quality of life among the elderly people. Neurology, Psychiatry and Brain Research. 2013; 9(1):48-52.

29- Foley D, Ancoli-Israel S, Britz P, Walsh J. Sleep disturbances and chronic disease in older adults results of the 2003 National Sleep Foundation Sleep in Americal Survey. Journal of Psychosomatic Research. 2004; 56: 497-502. 